\title{
Epidemiology of Brain Tumors in Qatar
}

\author{
Khaled Al-Sawalmeh ${ }^{1, *}$, Ghanem Al-Sulaiti' ${ }^{2}$, Ali Raza Ali ${ }^{2}$, Reem Sulaiman ${ }^{1}$ and Issam Al-Bozom ${ }^{1}$ \\ ${ }^{1}$ Department of Laboratory Medicine and Pathology, Hamad Medical Corporation, Doha, Qatar \\ ${ }^{2}$ Department of neurosurgery, Hamad Medical Corporation, Doha, Qatar
}

*Corresponding author: Khaled Al-Sawalmeh, Department of Laboratory Medicine and Pathology, Hamad Medical Corporation, Doha, Qatar, Tel: +97450246226; E-mail: kalsawalmeh@hamad.qa

Received: 11 May, 2020 | Accepted: 05 Jun, 2020 | Published: 10 Jun, 2020

Citation: Al-Sawalmeh K, Al-Sulaiti G, Ali AR, Sulaiman R, Al-Bozom I (2020) Epidemiology of Brain Tumors in Qatar. J Epidemiol Public Health Rev 5(2): dx.doi.org/10.16966/2471-8211.190

Copyright: (c) Al-Sawalmeh $\mathrm{K}$, et al. This is an open-access article distributed under the terms of the Creative Commons Attribution License, which permits unrestricted use, distribution, and reproduction in any medium, provided the original author and source are credited.

\begin{abstract}
Background: Brain tumors in general are rare tumors and their epidemiologic features in the Middle East including the state of Qatar is rarely tackled. The objective of this study is to delineate the epidemiologic characteristic of brain tumors in the gulf state of Qatar.

Methods: We run a retrospective analysis of brain tumors diagnosed histologically in the main Hospital in Qatar between January $1^{\text {st }}$, 2006-December $31^{\text {st }}$ 2015. We studied the following indices: Histologic type, gender, age, and location. We obtained the demographic data from Qatar Planning and Statistic Authority (www.psa.gov.qa).

Results: A total of 383 cases of primary brain tumors were identified and analyzed during the 10 years period studied. The incidence rate of primary brain tumors is $2.2 / 100,000$ population. Normalized Male/Female ratio is $0.93: 1$. The commonest histological subtypes were Meningioma (21.9\%) and Glioblastoma (18.3\%) followed by Astrocytoma (G2-G3) (18\%) and Oligodendroglioma (15.1\%).

Conclusion: Brain tumors incidence in the state of Qatar is low $(2.2 / 100,000)$ when compared to developed countries which may reflects the young age population.
\end{abstract}

Keywords: Epidemiology; Qatar; Prevalence; Meningioma; Glioblastoma; Primary brain tumors

\section{Introduction}

Brain tumors are relatively uncommon and account for about $2 \%$ of all cancers [1]. Brain tumors include benign and malignant types; they can arise from different cells of the central nervous system (CNS) or from their supportive structures. These tumors have different epidemiology between children and adults being rare in adults while in children they rank the second most common malignant tumor in the US [1]. There are wide differences in the epidemiology of brain tumors between countries in which they appear to be higher in more developed countries (males (M), 5.8 and females (F), 4.1 per 100,000 population) than in less developed countries (males 3.0 and females 2.1 per 100,000 population) [2]. Brain tumors are an important cause of morbidity and mortality in both adults and children with high burden to families and health care systems [3] in which for example, glioblastoma's 5 year survival rate is about 4.7 with no obvious role of surgery or radiotherapy as part of first-line treatment [4], so early recognition and management is essential for improving life quality and extending survival [5]. Accordingly, better understanding in the pathogenesis of these tumors is important for prevention and better management.

The state of Qatar is a small country in the Arabian (Persian) gulf. Due to discovery of natural resources, its population has grown rapidly from 1,043,000 in 2006 to almost 2.4 million in 2015 [6]. There were no attempts previously to describe the incidence of various brain tumors in Qatar. The goal of this study is to create a record of the epidemiology of primary brain tumors in Qatar. Although this is a hospital-based study, however, it is expected to reflect the whole Qatar population since pathology services are centralized at Hamad General Hospital and represent the entire state of Qatar.

\section{Material and Methods}

This was a retrospective study that was approved by IRB (Institute Review Board) and research center at Hamad Medical Corporation (Protocol ID: MRC-02-20-359). It was conducted on all cases diagnosed histologically as brain tumors (e.g., tumors originating from brain parenchyma, cranial nerves, meninges, pituitary gland, pineal gland, skull base and supportive structures) in the period between January 2006 and December 2015, based on the records of the Department of Pathology, Hamad General Hospital, Doha, Qatar, which is the only place of examination of all brain tumors in Qatar. The World Health Organization (WHO) classification of tumors of the central nervous system (2007) was used in this study. The following parameters were retrieved from patient's files: age at time of diagnosis, gender, time (year) of diagnosis, location, and histological type. We excluded the records that lacked histologic diagnosis and cases of brain metastasis. The mid-year population statistics provided 
by Qatar Planning and Statistics Authority from 2006-2015 was used in the calculation of crude incidence rate.

\section{Results}

A total of 415 cases of brain tumor were retrieved during the study period (2006-2015), of which 383 cases fulfilled our inclusion criteria and 32 cases were excluded as being classified as brain metastasis. See table 1 .

The overall incidence rate of primary brain tumors in Qatar was 2.2 per 100,000 population. However, it was variable during the study period, being highest in 2012 and lowest in 2011 (3.54 per 100,000 person years and 1.558 per 100,000 person years, respectively). See figures 1 and 2 .

Figure 3 shows wide variability of Crude Incidence Rate (CIR) as depicted by one year moving average with two high peaks in 2008 and 2012.

The percentage of pediatric tumors $(<20$ years of age) is $11.5 \%$, adult tumors (20-60 years of age) is $80.5 \%$, and geriatric tumors ( $>60$ years of age) is $8 \%$. See table 2 and figure 4 .

Most common primary brain tumors were in the supratentorial region, followed by infratentorial region (see Figure 5). Other locations include thalamus, multiple lesions, and few unknown location cases.

The male: female ratio of primary brain tumors was $2.8: 1$, however, due to the skewed population living in Qatar which has a M:F ratio of $3: 1$, and the adjusted male: female ratio is $0.93: 1$.

The most common brain tumor types in Qatar were meningioma (21.9\%) and glioblastoma (18.3\%) followed by astrocytoma (G2-G3) (18\%) and oligodendroglioma (15.1\%).
Glial tumors were more common in males while meningioma was more in females. See table 1.

\section{Discussion}

This is the first epidemiological study of primary brain tumors in Qatar.

The average crude incidence rate (CIR) of primary brain tumors in Qatar was noticed to be 2.2 per 100,000 person-year being lowest in 2007 ( 1.5 per 100,000 person-year) and highest in 2012 (3.5 per 100,000 person-year); and shows an increase in one year moving average (Figure 3 ) which can be partly explained by improving health care in Qatar and the improved availability of less invasive diagnostic tests. The CIR of primary brain tumors in the current study was marginally less than that in the other developing nations such as Iran, Saudi Arabia, Kuwait and Jordan (2.74,3.1,3.02 and 5.01 per 100,000 person-years, respectively) [7-10] while lower than developed counterparts such as South Korea and France (11.69 and 15.8 per 100,000 person-years, respectively) [11,12].

Individuals in the 31-50 age range had the highest percentage of $48 \%$, whereas patients within the 11-20 age categories had the lowest percentage of $2.6 \%$.

Overall, developed nations have higher primary brain tumors incidence than developing ones [2]. Some authors attribute such variations to socioeconomic factors since Western Europe, North America and Australia report higher incidence globally [13,14], whereas Eastern Africa ranks on the bottom of the affected regions [15]. These variations could also be justified by the demographic nature of each country and its unique age group distribution as older age groups witness higher incidence rate.

Table 1: Distribution of brain tumors in Qatar over ten years 2006-2016.

\begin{tabular}{|c|c|c|c|c|c|c|}
\hline Histologic description & \multicolumn{2}{|c|}{$M: F$} & Total & Percentage \% & M-F incidence ratio & $\begin{array}{l}\text { Normalized M-F } \\
\text { incidence ratio }\end{array}$ \\
\hline \multicolumn{7}{|l|}{ 1. Glial Tumors } \\
\hline Pilocytic tumors & 7 & 4 & 11 & 2.9 & $1.75: 1$ & $0.6: 1$ \\
\hline Astrocytic tumors (G 2-3) & 54 & 15 & 69 & 18 & $3.6: 1$ & $1.6: 1$ \\
\hline Glioblastoma & 62 & 8 & 70 & 18.3 & $7.75: 1$ & $2.6: 1$ \\
\hline Oligodendroglial tumors & 53 & 5 & 58 & 15.1 & $10.6: 1$ & $3.5: 1$ \\
\hline Mixed gliomas & 6 & 6 & 12 & 3.1 & $1: 1$ & $0.3: 1$ \\
\hline Ependymal tumors & 10 & 2 & 12 & 3.1 & 5.1 & $1.6: 1$ \\
\hline $\begin{array}{l}\text { Neuronal and mixed neuroglial } \\
\text { tumors }\end{array}$ & 7 & 2 & 9 & 2.4 & $3.5: 1$ & $1.16: 1$ \\
\hline \multicolumn{7}{|l|}{ 2. Non-Glial Tumors } \\
\hline Embryonal tumors & 16 & 7 & 23 & 6 & $2.3: 1$ & $0.76: 1$ \\
\hline Choroid plexus tumors & 0 & 2 & 2 & 0.5 & 0.2 & 0.2 \\
\hline Pineal tumors & 0 & 0 & 0 & 0 & & \\
\hline Meningeal tumors & 42 & 42 & 84 & 21.9 & $1: 1$ & $0.3: 1$ \\
\hline Germ cell tumors & 3 & 0 & 3 & 0.8 & $3: 0$ & 1:0 \\
\hline Tumors of sellar region & 7 & 3 & 10 & 2.6 & $2.3: 1$ & $0.76: 1$ \\
\hline $\begin{array}{l}\text { Lymphoma and other } \\
\text { hemopoietic lesions }\end{array}$ & 7 & 3 & 10 & 2.6 & $2.3: 1$ & $0.76: 1$ \\
\hline Mesenchymal tumors & 9 & 1 & 10 & 2.6 & 9:1 & $3: 1$ \\
\hline Total & 283 & 100 & 383 & 100 & $2.83: 1$ & 0.93:1 \\
\hline
\end{tabular}




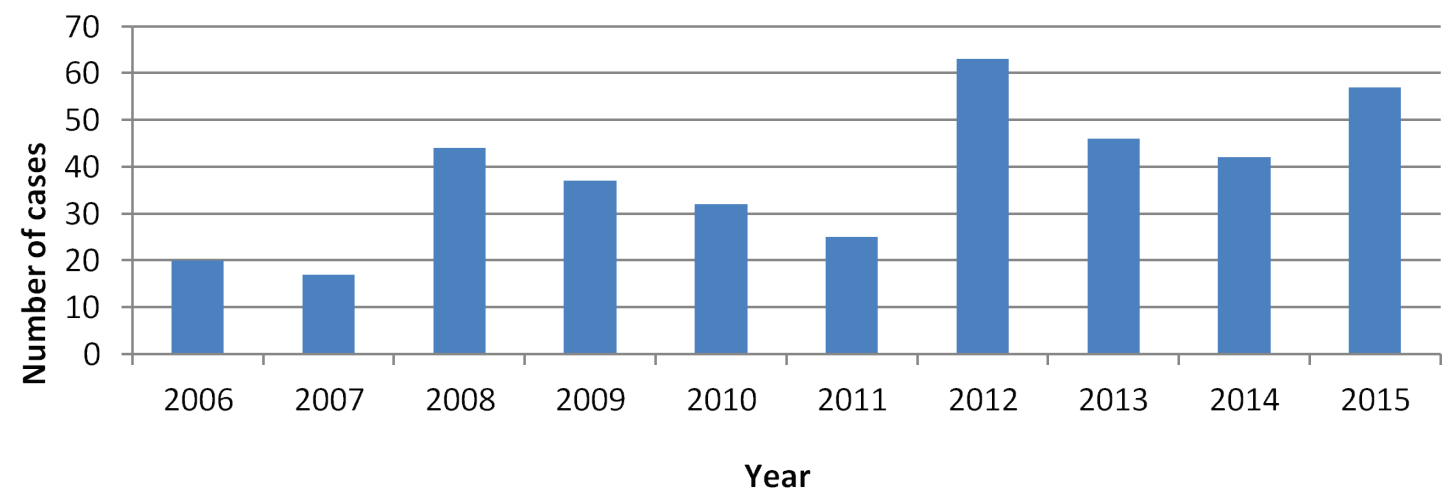

Figure 1: Frequency of primary CNS tumors in Qatar, 2006-2015.

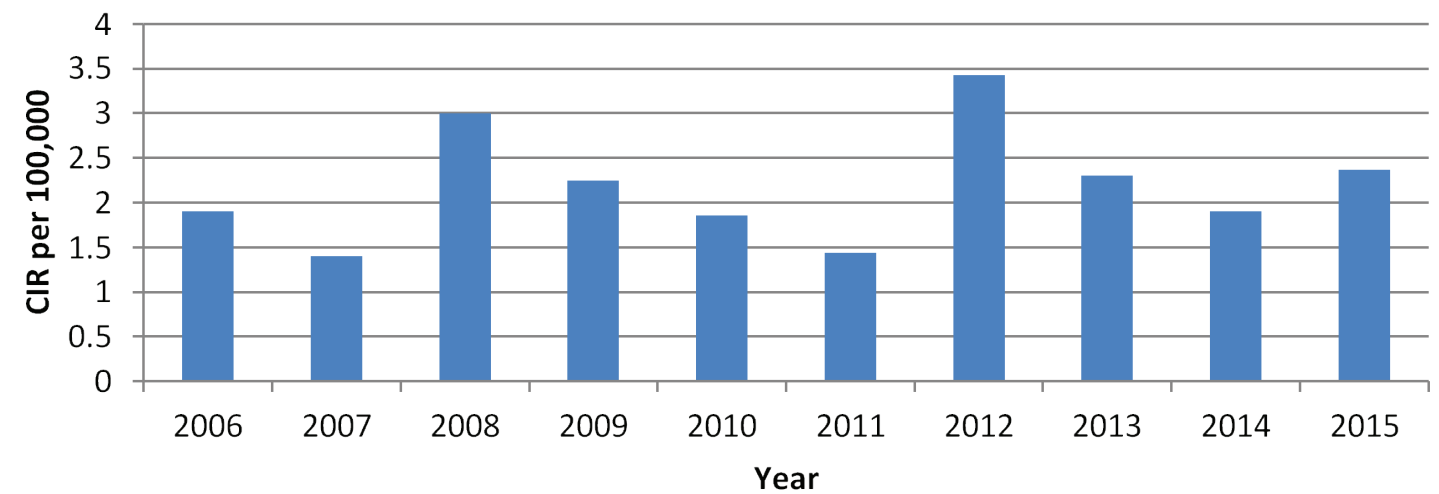

Figure 2: CIR per 100.000 primary CNS tumors in Qatar, 2006-2015.

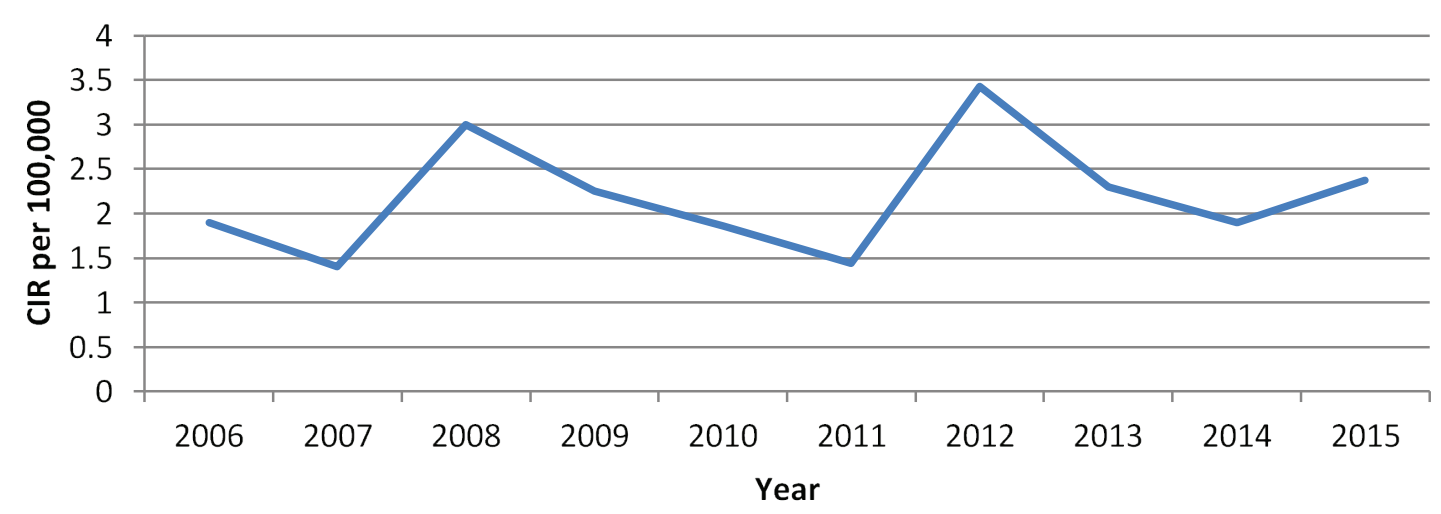

Figure 3: One year moving average of primary CNS tumors per 100.000 in Qatar, 2006-2015.

For example, according to midyear population, $15 \%$ of Qatar's population was under the age of 14 years, $83.9 \%$ was in the age range 15-64, and only $1.1 \%$ was above the age of 65 years [6]. These percentages were slightly similar to other developing countries such as Jordan and Iran (i.e., 34.95, 23.68\% under 14 years, $61.97 \%, 71.19 \%$ $15-64$ years, $3.44 \%, 5.2 \%$ respectively above 65 years) $[7,16]$ and visibly varied from other more developed countries such as the United States (i.e., $19.73 \%$ under 14 years, $66.94 \% 15-64$ years, and $13.3 \%$ above 65 years) [17] and South Korea (i.e., 15\% under 14 years, 72.83\% 15-64 years, and 11.44 above 65 years) [18]. This difference in incidence rate between developing and developed countries is partially related to age distribution of population.

The male: female ratio of primary brain tumors was $2.8: 1$, however, being the population skewed towards dominance of males living in Qatar due to the presence of expatriate male work force (M:F ratio of $3: 1$ ), the adjusted male:female ratio of primary brain tumors is $0.93: 1$; these results are comparable to other countries such as the United 
Table 2: Frequency of brain tumors as a function of population age strata.

\begin{tabular}{|c|c|c|c|c|c|c|c|c|}
\hline \multirow{2}{*}{ Histologic description } & \multicolumn{8}{|c|}{ Frequency in age strata } \\
\hline & $0-10$ & $11-20$ & $21-30$ & $31-40$ & $41-50$ & $51-60$ & $>60$ & Total \\
\hline \multicolumn{9}{|l|}{ 1. Glial Tumors } \\
\hline Pilocytic tumors & 9 & 0 & 1 & 0 & 1 & 0 & 0 & 11 \\
\hline Astrocytic tumors (G 2-3) & 2 & 4 & 16 & 23 & 15 & 7 & 2 & 69 \\
\hline Glioblastoma & 1 & 1 & 10 & 10 & 20 & 19 & 9 & 70 \\
\hline Oligodendroglial tumors & 0 & 1 & 15 & 22 & 12 & 4 & 4 & 58 \\
\hline Mixed gliomas & 0 & 0 & 3 & 6 & 2 & 0 & 1 & 12 \\
\hline Ependymal tumors & 2 & 0 & 3 & 5 & 0 & 2 & 0 & 12 \\
\hline Neuronal and mixed neuroglial tumors & 1 & 2 & 0 & 1 & 3 & 2 & 0 & 9 \\
\hline \multicolumn{9}{|l|}{ 2. Non-Glial Tumors } \\
\hline Embryonal tumors & 16 & 1 & 1 & 4 & 0 & 1 & 0 & 23 \\
\hline Choroid plexus tumors & 2 & 0 & 0 & 0 & 0 & 0 & 0 & 2 \\
\hline Pineal tumors & 0 & 0 & 0 & 0 & 0 & 0 & 0 & 0 \\
\hline Meningeal tumors & 0 & 0 & 7 & 15 & 32 & 20 & 10 & 84 \\
\hline Germ cell tumors & 0 & 0 & 3 & 0 & 0 & 0 & 0 & 3 \\
\hline Tumors of sellar region & 0 & 1 & 2 & 4 & 1 & 1 & 1 & 10 \\
\hline $\begin{array}{l}\text { Lymphoma and other hemopoietic } \\
\text { lesions }\end{array}$ & 0 & 0 & 0 & 1 & 3 & 3 & 3 & 10 \\
\hline Mesenchymal tumors & 1 & 0 & 5 & 2 & 2 & 0 & 0 & 10 \\
\hline Total & 34 & 10 & 66 & 93 & 91 & 59 & 30 & 383 \\
\hline
\end{tabular}

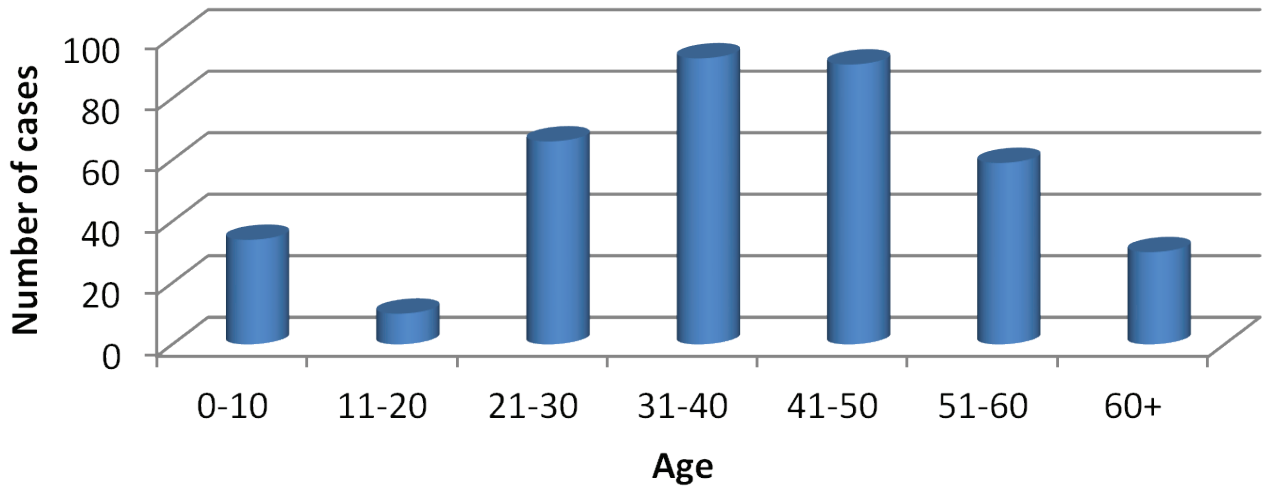

Figure 4: Frequency of CNS tumors by age, Qatar 2006-2015.

States, France, South Korea and Jordan (i.e., 0.73, 0.86, 0.69 and 0.85 respectively) $[7,12,19]$. However, primary brain tumors appear to be more frequent in males than in females worldwide (i.e., male to female ratio is 1.42$)$ [2].

The most common brain tumor types in Qatar were meningioma $(21.9 \%)$ and glioblastoma (18.3\%). These findings were comparable to other regional developing nations such as Jordan (e.g., meningioma (26.2\%), glioblastoma (18.9\%) [7] and Iran (e.g., meningioma (27.8\%), glioblastoma (13.8\%)) [10], but visibly varied from developed countries such as the United States (e.g., meningioma (36.1\%), glioblastoma (15.7 $\%$ ) [19] and South Korea (e.g., meningioma (31.1\%), glioblastoma $(36.5 \%))[11]$.
One limitation of this study is that the diagnosis rendered were based on 2007 WHO classification since the time period of the cases studied was before 2016, the date at which the new classification of brain tumors which incorporate molecular data in the pathological diagnosis "Integrated diagnoses" which carries more details of behavior as well as prognostic features of brain tumors.

The new integrated diagnosis might affect the epidemiologic evaluation of future brain tumors cases taken into consideration that integrated diagnosis doesn't depend only on morphology but adding to it molecular sub typing of the tumor, which further sub classify brain tumor from just morphology alone. A more extending work for better and more accurate sub classification of brain tumors is essential and not dependent only on morphology. 


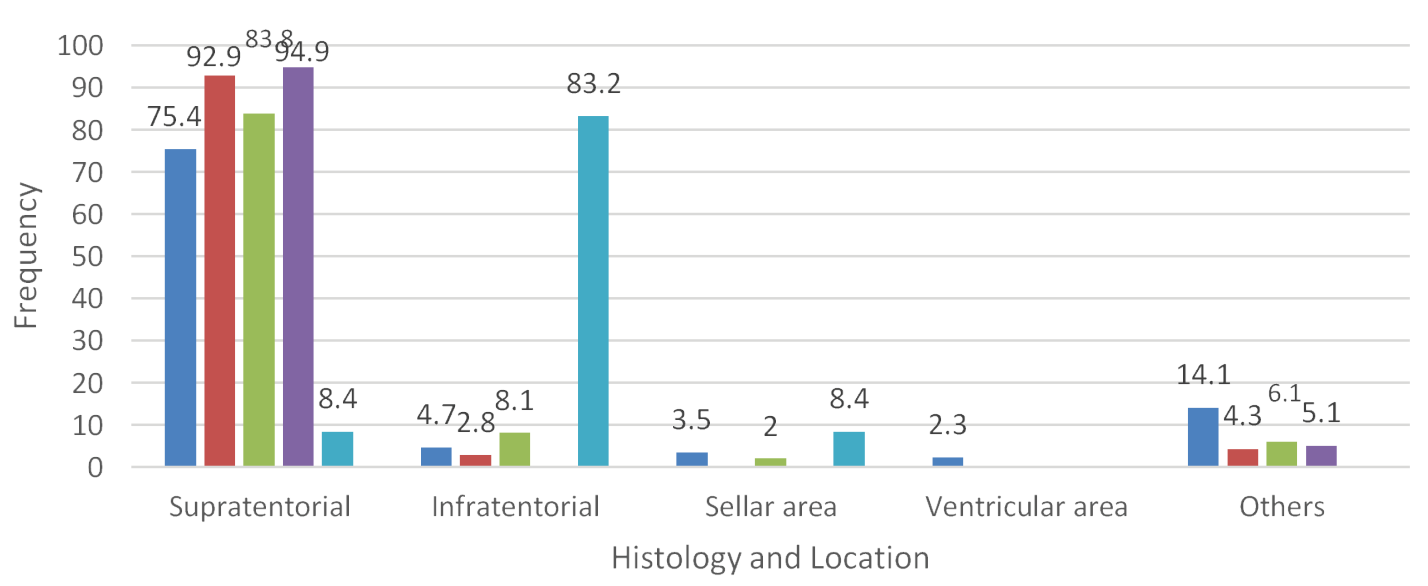
- Meningeal Tumors
- Glioblastoma
Astrocytic Tumors(G 2-3)
- Oligodendroglial Tumors a Pilocytic tumors

Figure 5: Frequency of common CNS tumors by location, Qatar 2006-2015.

\section{Conclusion}

The results suggest that epidemiology of primary brain tumors in Qatar is comparable to middle eastern and developing countries like Jordan, Kuwait, Saudi Arabia, and Iran and different when compared to developed countries like United States, South Korea, and France. The incidence of primary brain tumors in Qatar is relatively low. The most common age affected is the young adult population taking into consideration that $83.9 \%$ of Qatar's population is of young age, it is more common in females after adjusting the skewed population ratio and Meningioma and Glioblastoma are the most common between primary brain tumors. Finally, it is necessary to expand the national Cancer Registry to include all brain tumors classified under 2016 WHO classification which predicts tumor biological behavior, hence facilitating population-based studies and enhancement of surveillance and planning.

\section{References}

1. Siegel RL, Miller KD, Jemal A (2019) Cancer statistics, 2019. CA Cancer J Clin 69: 7-34.

2. Bondy ML, Scheurer ME, Malmer B, Barnholtz-Sloan JS, Davis FG, et al. (2008) Brain Tumor Epidemiology: Consensus from the Brain Tumor Epidemiology Consortium. Cancer 113: 1953-1968.

3. de Robles P, Fiest KM, Frolkis AD, Pringsheim T, Atta C, et al. (2015) The Worldwide Incidence and Prevalence of Primary Brain Tumors: A Systematic Review and Meta-Analysis. Neuro Oncol 17: 776-783.

4. Omuro A, DeAngelis LM (2013) Glioblastoma and Other Malignant Gliomas: A Clinical Review. JAMA 310: 1842-1850.

5. Lacy J, Saadati H, Yu JB (2012) Complications of Brain Tumors and Their Treatment. Hematol Oncol Clin North Am 26: 779-796.

6. From data collected by Ministry of Development Planning and Statistics.

7. Tamimi AF, Tamimi I, Abdelaziz M, Saleh Q, Obeidat F, et al. (2015) Epidemiology of Malignant and Non-Malignant Primary Brain Tumors in Jordan. Neuroepidemiology 45: 100-108.
8. Katchy KC, Alexander S, Al-Nashmi NM, Al-Ramadan A (2013) Epidemiology of Primary Brain Tumors in Childhood and Adolescence in Kuwait. Springerplus 2: 58.

9. Ibrahim AWM (1992) C.N.S. Tumors in Eastern Saudi Arabia. Neurosurg Rev 15: 295-302.

10. Jazayeri SB, Rahimi-Movaghar V, Shokraneh F, Saadat S, Ramezani R (2013) Epidemiology of Primary CNS Tumors in Iran: A Systematic Review. Asian Pac J Cancer Prev 14: 3979-3985.

11. Lee $\mathrm{CH}$, Jung KW, Yoo H, Park S, Lee SH (2010) Epidemiology of Primary Brain and Central Nervous System Tumors in Korea. J Korean Neurosurg Soc 48: 145-152.

12. Bauchet L, Rigau V, Mathieu-Daudé $H$, Figarella-Branger $D$, Hugues D, et al. (2007) French Brain Tumor Data Bank: Methodology and First Results on 10,000 Cases. J Neurooncol 84: 189-199.

13. Deltour I, Johansen $C$, Auvinen A, Feychting $M$, Klaeboe $L$, et al. (2009) Time Trends in Brain Tumor Incidence Rates in Denmark, Finland, Norway, and Sweden, 1974-2003. J Natl Cancer Inst 101: 1721-1724.

14. Dobes M, Shadbolt B, Khurana VG, Jain S, Smith SF, et al. (2011) A Multicenter Study of Primary Brain Tumor Incidence in Australia (2000-2008). Neuro Oncol 13: 783-790.

15. Ferlay J, Shin HR, Bray F, Forman D, Mathers C, et al. (2010) Estimates of Worldwide Burden of Cancer in 2008: GLOBOCAN 2008. Int J Cancer 127: 2893-2917.

16. World Bank (2015) Iran: age Structure from 2008 to 2018. Statista.

17. World Bank (2015) Age Distribution in the United States from 2008 to 2018. Statista.

18. World Bank (2015) South Korea: age Structure between 2008 and 2018. Statista.

19. Ostrom QT, Gittleman H, Liao P, Rouse C, Chen Y, et al. (2014) CBTRUS Statistical Report: Primary Brain and Central Nervous System Tumors Diagnosed in the United States in 2007-2011. Neuro Oncol 16: iv1-iv63. 